\title{
How do primary care physicians in Singapore keep healthy?
}

Ngiap Chuan $\underline{T a n}^{1}$, MMed, MCI, Lily ${\underline{A w^{2}}}^{2}$ MBBS, FCFPS, Lay Wai Khin ${ }^{3}$, MBBS, MSc, Thamotharampillai $\underline{\text { Thirumoorthy }}{ }^{4}$, FRCP, FAMS, Shih Hui Lim ${ }^{4,5}$, FRCP, FAMS, Bee Choo $\underline{T a i}^{6}$, BA, PhD, Lee Gan $\underline{G o h}^{7}$, FCFP, FRACGP

INTRODUCTION Not much is known regarding how primary care physicians (PCPs) in Singapore keep themselves healthy and mitigate ill health. This study aims to determine the health-seeking behaviour of local PCPS and to identify the predictors of local PCPs attaining the recommended level of exercise.

METHODS This study was a cross-sectional questionnaire survey, which included questions on the demographic characteristics, practice profiles and health-seeking behaviour of PCPs. The sampling frame was the 1,400 listed members of the College of Family Physicians Singapore. The anonymised survey was executed in two phases: a postal survey, followed by a web-based survey on the College of Family Physicians Singapore website. The two data sets were collated; the categorical variables, summarised; and the differences between subgroups (based on exercise engagement), compared using Fisher's exact test. The effect of each risk factor on exercise duration was quantified using odds ratio (OR) estimate and $95 \%$ confidence interval $(\mathrm{Cl})$. Multivariate logistic regression analysis was performed to identify significant predictors of exercise engagement.

RESULTS A total of 631 PCPs participated in the survey $-26 \%$ were $\leq 34$ years old, $58 \%$ were male, $21 \%$ were single, $34 \%$ were singleton practitioners, and $56 \%$ were private practitioners. The percentage of PCPs who exercised $\geq 2.5$ hours weekly was $29 \%$, while $28 \%$ exercised $<0.5$ hours weekly. Of the PCPs surveyed, $1 \%$ currently smoke, $0.8 \%$ drink more than 14 units of alcohol weekly, $60 \%$ undertook health screening, $65 \%$ had blood investigations done, and $64 \%$ had taken preventive measures such as getting influenza vaccination.

CONCLUSION While local PCPs generally did not have undesirable habits such as smoking and alcohol abuse, they could further increase their exercise intensity and undertake more preventive measures such as getting vaccinated against various diseases.

Keywords: exercise, health, primary care physicians, vaccination

\section{INTRODUCTION}

Doctors are typically perceived to be healthier than the general population. In fact, an Australian study reported that doctors have a lower mortality rate. ${ }^{(1)}$ Nonetheless, the prevalence of chronic diseases among doctors is similar to that of the general population. (2) Primary care physicians (PCPs) have often assumed the role of counsellors, educating patients on the importance of adopting healthy lifestyles that include regular exercise. Regular physical activity and exercise have been shown to reduce the morbidity and mortality from many chronic diseases, and are therefore vital for the health and well-being of people of all ages. Regular physical activity has been shown to reduce the risk of developing or dying from heart disease, diabetes mellitus, colonic cancer and hypertension. On average, people who are physically active outlive those who are not. However, little is known regarding how PCPs 'walk the talk' and whether they keep themselves healthy with regular physical activity to mitigate premature development of chronic diseases.

In Singapore, PCPs in public and private primary care clinics work different hours due to Singapore's dual primary healthcare system. Groups of doctors work in public polyclinics, which operate during office hours from Mondays to Saturdays. However, general practitioners (GPs) in private clinics (singleton or group practices) typically provide primary care service in the evenings, with some even operating round-theclock. Locums are employed to relieve these doctors when they have other commitments. In other words, private GPs are more likely to work irregular hours than doctors who work in public polyclinics. With this variety of working conditions, access to a healthy lifestyle may differ across different groups of PCPs. Thus, this study aimed to determine the health-seeking behaviour of PCPs in Singapore and to identify the predictors of PCPs attaining the recommended level of exercise.

\section{METHODS}

As there was no validated survey instrument in Singapore to determine the health and illness behaviour of PCPs, we developed the 'Dr4Dr' questionnaire based on personal experience, literature search and feedback from fellow doctors in the community. The questionnaire covers the participants'

\footnotetext{
${ }^{1}$ SingHealth Polyclinics - Department of Research, ${ }^{2}$ Lily Aw Pasir Ris Family Clinic \& Surgery, ${ }^{3}$ Investigational Medicine Unit, Yong Loo Lin School of Medicine, National University Hospital System, ${ }^{4}$ Duke-NUS Graduate Medical School, ${ }^{5}$ Academy of Medicine, ${ }^{6}$ Saw Swee Hock School of Public Health, National University of Singapore, ${ }^{7}$ Division of Family Medicine, National University Health System, Singapore

Correspondence: Dr Tan Ngiap Chuan, Director, SingHealth Polyclinics - Department of Research, 167 Jalan Bukit Merah, \#15-10, Tower 5, Connection One, Singapore 150167. Tan.Ngiap.Chuan@singhealth.com.sg
} 
Table I. Demographic and practice profiles of the primary care physicians (PCPs), according to their reported duration of exercise.

\begin{tabular}{|c|c|c|c|c|c|}
\hline \multirow[t]{3}{*}{ Variable } & \multicolumn{3}{|c|}{ No. (\%) } & \multirow{3}{*}{$\begin{array}{l}\text { Unadjusted } \\
\text { OR }(95 \% \mathrm{CI})\end{array}$} & \multirow[t]{3}{*}{ p-value } \\
\hline & \multirow{2}{*}{$\begin{array}{c}\text { Total } \\
(n=631)\end{array}$} & \multicolumn{2}{|c|}{ Exercise (hrs/wk) } & & \\
\hline & & $<2.5(n=451)$ & $\geq 2.5(n=180)$ & & \\
\hline \multicolumn{6}{|l|}{ Age group (yrs) } \\
\hline $25-34$ & $163(25.8)$ & $130(28.8)$ & $33(18.3)$ & 1.00 & - \\
\hline $35-44$ & $207(32.8)$ & $150(33.3)$ & $57(31.7)$ & $1.49(0.92-2.44)$ & 0.106 \\
\hline $45-54$ & $160(25.4)$ & 108 (23.9) & $52(28.9)$ & $1.89(1.14-3.14)$ & 0.013 \\
\hline $55-64$ & $66(10.5)$ & $40(8.9)$ & $26(14.4)$ & $2.56(1.37-4.78)$ & 0.003 \\
\hline$\geq 65$ & $35(5.5)$ & $23(5.1)$ & $12(6.7)$ & $2.06(0.93-4.55)$ & 0.076 \\
\hline \multicolumn{6}{|l|}{ Gender } \\
\hline Female & $265(42.1)$ & $206(45.8)$ & $59(32.8)$ & 1.00 & - \\
\hline Male & $365(57.9)$ & $244(54.2)$ & $121(67.2)$ & $1.73(1.21-2.49)$ & 0.003 \\
\hline \multicolumn{6}{|l|}{ Marital status } \\
\hline Single & $130(20.6)$ & $78(17.3)$ & $52(28.9)$ & 1.00 & - \\
\hline Married/divorced/widowed/separated & $501(79.4)$ & $373(82.7)$ & $128(71.1)$ & $0.52(0.34-0.77)$ & 0.001 \\
\hline \multicolumn{6}{|l|}{ Ownership of practice } \\
\hline Private clinic & $318(55.9)$ & $218(53.6)$ & $100(61.7)$ & 1.00 & - \\
\hline Public primary care clinic/institution* & $251(44.1)$ & $189(46.4)$ & $62(38.3)$ & $0.72(0.49-1.04)$ & 0.007 \\
\hline \multicolumn{6}{|l|}{ Operational mode of practice } \\
\hline Singleton & $199(34.0)$ & 139 (33.3) & $60(35.7)$ & 1.00 & - \\
\hline Group & $387(66.0)$ & $279(66.7)$ & $108(64.3)$ & $0.90(0.62-1.31)$ & 0.571 \\
\hline \multicolumn{6}{|l|}{ Professional profile } \\
\hline PCP & $590(97.5)$ & $418(97.4)$ & $172(97.7)$ & 1.00 & - \\
\hline Specialist practising as PCP & $15(2.5)$ & $11(2.6)$ & $4(2.3)$ & $0.88(0.28-2.81)$ & 0.834 \\
\hline \multicolumn{6}{|l|}{ No. of working hours } \\
\hline$<25$ & $70(11.2)$ & $50(11.2)$ & $20(11.2)$ & 1.00 & - \\
\hline $20-50$ & $408(65.2)$ & $297(66.3)$ & $111(62.4)$ & $0.93(0.53-1.64)$ & 0.813 \\
\hline$\geq 25$ & $148(23.6)$ & $101(22.5)$ & $47(26.4)$ & $1.16(0.62-2.17)$ & 0.634 \\
\hline Locums (full-/part-time) & $144(22.8)$ & $106(23.5)$ & $38(21.1)$ & $0.88(0.58-1.34)$ & 0.562 \\
\hline
\end{tabular}

Note: Figures for some items do not add up to total due to missing data. ${ }^{*}$ Includes family medicine departments in universities or selected restructured hospitals. $\mathrm{Cl}$ : confidence interval; OR: odds ratio

demographic characteristics, practice profiles, health and illness behaviours and their views of a proposed healthcare support system for doctors in Singapore.

Participants in the present study were PCPs working in various types of primary care practices, including public and private primary care clinics, as well as family medicine units within the academic departments of the two medical schools and hospitals in Singapore. The sampling frame was the College of Family Physicians Singapore (CFPS) membership list, which consisted of 1,400 members as at June 2011. Of the 1,400 members, $558(40 \%)$ were female doctors while $842(60 \%)$ were male doctors; 811 (58\%) were < 45 years of age and $589(42 \%)$ were $\geq 45$ years of age.

To increase the response rate, the questionnaire was administered in two phases - Phase A and Phase B. In Phase A, printed copies of the questionnaire were mailed to the participants and some were fielded to them during a family medicine training module organised by CFPS. In the mailed survey, stamped envelopes addressed to CFPS were provided for participants to return the completed questionnaire. In Phase B, a web-based survey using the same questionnaire was carried out via the CFPS website. The SurveyMonkey software (SurveyMonkey, Palo Alto, CA, USA), which allows only a single entry from any one email address, was used. These two approaches were used to allow greater access and convenience for the PCPs to self-administer the questionnaire. To ensure truthful responses to the questions and prevent any potential stigmatisation, the questionnaire was anonymised. All data obtained from Phases A and $B$ was transcribed into a Microsoft Excel file (Microsoft Corp, Redmond, WA, USA). The study was approved by the Institutional Review Board of the National University of Singapore.

Categorical variables were summarised using frequencies and percentages, and differences between subgroups (categorised according to duration of exercise engagement) were compared using Fisher's exact test. The effect of each factor on exercise duration was quantified using odds ratio (OR) estimate and its associated 95\% confidence interval $(\mathrm{Cl})$. To identify independent predictors of exercise engagement, account for putative confounders, and prevent potentially important factors from being missed out, variables with a $p$-value of $\leq 0.1$ in the bivariate analysis were further considered for inclusion in the multivariate logistic regression model. Model selection was carried out based on the likelihood ratio test. All statistical analyses were performed using STATA software version 12.0 (StataCorp, College Station, TX, USA), assuming a two-sided test at the conventional 0.05 level of significance.

\section{RESULTS}

A total of 631 PCPs participated in the survey, resulting in a response rate of $45.1 \%$. The demographic and practice profiles of the participants are shown in Table I. Junior doctors (i.e. 
Table II. Details on the lifestyles of the primary care physicians, according to their reported duration of exercise.

\begin{tabular}{|c|c|c|c|c|c|}
\hline \multirow[t]{3}{*}{ Variable } & \multicolumn{3}{|c|}{ No. (\%) } & \multirow{3}{*}{$\begin{array}{l}\text { Unadjusted } \\
\text { OR }(95 \% \mathrm{CI})\end{array}$} & \multirow[t]{3}{*}{ p-value } \\
\hline & \multirow{2}{*}{$\begin{array}{c}\text { Total } \\
(n=631)\end{array}$} & \multicolumn{2}{|c|}{ Exercise (hrs/wk) } & & \\
\hline & & $<2.5(n=451)$ & $\geq 2.5(n=180)$ & & \\
\hline \multicolumn{6}{|l|}{ Smoking status } \\
\hline Never & $596(94.5)$ & $423(93.8)$ & $173(96.1)$ & 1.00 & - \\
\hline Ex-smoker & $28(4.4)$ & $22(4.9)$ & $6(3.3)$ & $0.67(0.27-1.67)$ & 0.388 \\
\hline Current smoker & $7(1.1)$ & $6(1.3)$ & $1(0.6)$ & $0.41(0.05-3.41)$ & 0.408 \\
\hline \multicolumn{6}{|c|}{ Weekly alcohol intake } \\
\hline Never & $304(48.2)$ & $221(49.0)$ & $83(46.1)$ & 1.00 & - \\
\hline$<7$ units & $306(48.5)$ & $220(48.8)$ & $86(47.8)$ & $1.04(0.73-1.48)$ & 0.825 \\
\hline $7-14$ units & $16(2.5)$ & $6(1.3)$ & $10(5.6)$ & $4.44(1.56-12.59)$ & 0.005 \\
\hline$>14$ units & $5(0.8)$ & $4(0.9)$ & $1(0.6)$ & $0.67(0.07-6.04)$ & 0.718 \\
\hline
\end{tabular}

Cl: confidence interval; OR: odds ratio

Table III. Preventive measures undertaken by the primary care physicians in the past one year, according to their reported duration of exercise.

\begin{tabular}{|c|c|c|c|c|c|}
\hline \multirow[t]{3}{*}{ Variable } & \multicolumn{3}{|c|}{ No. (\%) } & \multirow{3}{*}{$\begin{array}{l}\text { Unadjusted } \\
\text { OR }(95 \% \mathrm{CI})\end{array}$} & \multirow[t]{3}{*}{ p-value } \\
\hline & \multirow{2}{*}{$\begin{array}{c}\text { Total } \\
(n=631)\end{array}$} & \multicolumn{2}{|c|}{ Exercise (hrs/wk) } & & \\
\hline & & $<2.5(n=451)$ & $\geq 2.5(n=180)$ & & \\
\hline Health screening & $380(60.2)$ & $248(55.0)$ & $132(73.3)$ & $2.25(1.54-3.29)$ & $<0.001$ \\
\hline Blood investigation* & $410(65.0)$ & $276(61.2)$ & $134(74.4)$ & $1.85(1.26-2.71)$ & 0.002 \\
\hline Urine test & $176(27.9)$ & 108 (23.9) & $68(37.8)$ & $1.93(1.33-2.79)$ & 0.001 \\
\hline Electrocardiography & $107(17.0)$ & $66(14.6)$ & $41(22.8)$ & $1.72(1.11-2.66)$ & 0.015 \\
\hline Any form of radiography ${ }^{+}$ & $103(16.3)$ & $66(14.6)$ & $37(20.6)$ & $1.51(0.97-2.36)$ & 0.069 \\
\hline Any form of imaging ${ }^{\ddagger}$ & $70(11.1)$ & $47(10.4)$ & $23(12.8)$ & $1.26(0.74-2.14)$ & 0.395 \\
\hline Any form of ultrasonography & $89(14.1)$ & $63(14.0)$ & $26(14.4)$ & $1.04(0.63-1.70)$ & 0.877 \\
\hline Any form of endoscopy & $46(7.3)$ & $33(7.3)$ & $13(7.2)$ & $0.99(0.51-1.92)$ & 0.967 \\
\hline Exercise treadmill test & $32(5.1)$ & $21(4.7)$ & $11(6.1)$ & $1.33(0.63-2.82)$ & 0.453 \\
\hline $\begin{array}{l}\text { Self-initiation of any of the } \\
\text { listed investigations" }\end{array}$ & $299(67.0)$ & $201(65.3)$ & $98(71.0)$ & $0.77(0.50-1.19)$ & 0.233 \\
\hline Vaccination against infectious diseases" & $380(64.2)$ & $263(61.9)$ & $117(70.1)$ & $0.69(0.47-1.02)$ & 0.063 \\
\hline
\end{tabular}

${ }^{*}$ Blood investigation may be part of a screening or diagnostic test (e.g. full blood count, lipid profile and thyroid function test). ${ }^{\dagger}$ Examples include ches radiography and kidneys, ureters and bladder radiography. ${ }^{\ddagger}$ Examples include computed tomography and magnetic resonance imaging. ${ }^{\S}$ Examples include myocardial perfusion imaging and two-dimensional echocardiography. "Data was not available for 185 participants for the category 'self initiation of the listed investigations'. 39 participants did not provide information on vaccination against infectious diseases. Calculation of the percentages, and statistical analysis, were done with the available data. $\mathrm{Cl}$ : confidence interval; OR: odds ratio

doctors aged $25-34$ years) made up $25.8 \%$ of the participants, while only $5.5 \%$ of participants were of retirement age ( $\geq 65$ years). More than half $(57.9 \%)$ of the participants were male, one-fifth (20.6\%) were single, more than half were $(55.9 \%)$ private GPs, and approximately one-third (34.0\%) were singleton practitioners. Only a small proportion $(2.5 \%)$ of specialists practised as PCPs. Among the 631 PCPs surveyed, only $28.5 \%$ of participants exercised at least 2.5 hours per week, as recommended by our local health authority; $28.4 \%$ of them exercised less than 0.5 hour per week. Only $1 \%$ of the participants were current smokers and $0.8 \%$ of them drank alcohol in excess of 14 units weekly (Table II).

Table III lists the preventive measures (e.g. laboratory investigations and vaccinations) that the PCPs undertook in the past year. Of the PCPs surveyed, $60.2 \%$ of them had undergone some form of health screening, $65.0 \%$ had undergone some form of blood investigation, and $67.0 \%$ had initiated the undertaking of these investigations (any listed in Table III) themselves. The percentage of PCPs that had taken preventive measures against infectious diseases (e.g. getting vaccinated against influenza) was $64.2 \%$.

Table IV shows the results of the multivariate logistic regression analysis used to determine the predictors of PCPs attaining $\geq 2.5$ hours of exercise per week. We found that PCPs who were older (especially between 45-64 years of age), married, consumed 7-14 units of alcohol (i.e. moderate intake) and had some form of advanced cardiac imaging, were more likely to attain the recommended level of exercise.

\section{DISCUSSION}

The present study is the first study to provide insight into the health-seeking behaviour of PCPs in Singapore. A healthy healthcare workforce, which includes PCPs, is fundamental in the health management of the general population.

In the present study, we found that approximately three out of ten PCPs (29\%) had adequate exercise of at least 150 mins per 
Table IV. Results of the multivariate logistic regression used to identify predictors of engagement in $\geq \mathbf{2 . 5}$ hours of exercise per week.

\begin{tabular}{|c|c|c|c|}
\hline Variable & Adjusted OR & $95 \% \mathrm{Cl}$ & p-value \\
\hline \multicolumn{4}{|l|}{ Age group (yrs) } \\
\hline $25-34$ & 1.00 & - & - \\
\hline $35-44$ & 2.18 & $1.23-3.87$ & 0.008 \\
\hline $45-54$ & 2.80 & $1.53-5.10$ & 0.001 \\
\hline $55-64$ & 3.45 & $1.66-7.17$ & 0.001 \\
\hline$\geq 65$ & 3.20 & $1.31-7.84$ & 0.011 \\
\hline \multicolumn{4}{|l|}{ Marital status } \\
\hline Single & 1.00 & - & - \\
\hline $\begin{array}{l}\text { Married/divorced/ } \\
\text { widowed/separated }\end{array}$ & 3.76 & $2.28-6.21$ & $<0.001$ \\
\hline \multicolumn{4}{|l|}{ Weekly alcohol intake } \\
\hline Never & 1.00 & - & - \\
\hline$<7$ units & 1.13 & $0.78-1.65$ & 0.515 \\
\hline $7-14$ units & 5.66 & $1.91-16.78$ & 0.002 \\
\hline$>14$ units & 0.71 & $0.07-6.86$ & 0.765 \\
\hline \multicolumn{4}{|l|}{ Health screening } \\
\hline No & 1.00 & - & - \\
\hline Yes & 2.15 & $1.42-3.26$ & $<0.001$ \\
\hline \multicolumn{4}{|l|}{$\begin{array}{l}\text { Any form of advanced } \\
\text { cardiac imaging }\end{array}$} \\
\hline No & 1.00 & - & - \\
\hline Yes & 3.67 & $1.37-9.85$ & 0.010 \\
\hline
\end{tabular}

week. According to the Health Promotion Board of Singapore, this is the minimal amount of exercise that will result in significant benefits to health, including a $20 \%-50 \%$ risk reduction of cardiovascular diseases (e.g. hypertension and stroke), type 2 diabetes mellitus, breast and colonic cancers, and dementia. ${ }^{(3)}$ The proportion of PCPs who had adequate exercise in the present study was noticeably lower than that reported in a British survey by Allibone et al. ${ }^{(4)}$ In that British survey, the authors found that $52 \%$ of the male GPs and $34 \%$ of the female GPs exercised vigorously. ${ }^{(4)}$

We found that $28 \%$ of the PCPs in the present study had little exercise, while the percentage of the general population (aged 18-69 years) with little exercise was 54\% according to the Singapore National Health Survey (NHS); ${ }^{(5)}$ however, the classification of the amount of exercise differed slightly between the two studies. In the NHS, commonly cited reasons for physical inactivity were inadequate time due to work and family commitments $(58 \%)$, laziness $(13 \%)$, and lack of exercise $(8 \%)$. In the present study, however, low levels of exercise was not related to the working hours of the PCPs. Further studies are thus needed to examine the barriers PCPs encounter in attaining regular exercise.

The PCPs in our study who achieved the recommended level of weekly exercise were married and more advanced in age. Both of these factors are inter-related as the propensity to get married increases with age. The prevalence of chronic diseases also increases with age, and this may explain the tendency of older PCPs to exercise regularly and undertake health screenings, in an effort to mitigate the risk of developing chronic diseases. We postulate that PCPs with known cardiovascular risks are more likely to evaluate their cardiac health with investigations such as cardiac imaging; they would likely also be more proactive in attempts to reduce their risks by exercising regularly. The subset of PCPs who achieved the recommended level of weekly exercise was also found to practise other healthy pursuits, such as moderate consumption of alcohol such as red wine, which is widely perceived to avert cardiovascular risks.

The smoking rate among PCPs varies from country to country. The proportion of PCPs in the present study who currently smoke $(1 \%)$ is far lower than the prevalence of current smokers in the general population (14\%). This smoking rate is comparable to a trend observed in developed countries such as the United States, Australia and New Zealand, where a steady decline in the smoking rate among physicians has been observed over the past 30 years. ${ }^{(6)}$ However, another study reported that almost a third (29\%) of GPs in France were current smokers. ${ }^{(7)}$ In the present study, the proportions of PCPs who were regular consumers of alcohol (3\%) and nonalcoholic drinks (48\%) are comparable to those of the general population (3\% regular alcoholic drinkers and 54\% nonalcoholic drinkers). ${ }^{(5)}$

We found that $64 \%$ of PCPs were vaccinated against infectious diseases such as influenza. This percentage is comparable to that of another survey, in which $69 \%$ of participating physicians were found to be vaccinated; the latter survey, however, was carried out in two tertiary care teaching hospitals in the United States. ${ }^{(8)}$ Healthcare workers are important reservoirs of transmission in hospitals, contributing to the morbidities and mortalities of patients, especially vulnerable groups of patients such as children and elderly patients. Thus, it is critical for PCPs to be vaccinated to reduce such transmissions. This is especially pertinent as upper respiratory tract infection remains one of the top five reasons for consultations in private and public primary care clinics in Singapore. ${ }^{(9)}$ The Centres for Disease Control and Prevention in the United States recommends that all healthcare workers receive annual influenza vaccination. ${ }^{(10)}$ Many studies have been conducted to determine the barriers encountered by healthcare workers in getting vaccinated. ${ }^{(8,11)}$ Lack of time, perceived uncertainty over vaccine efficacy and fear of side effects were often quoted as primary reasons for declining vaccination. ${ }^{(12)}$ A greater knowledge about vaccines is associated with a higher vaccination rate.

In the present study, the proportions of PCPs that had some form of health screening and blood investigations carried out in the past one year were $60 \%$ and $65 \%$, respectively. This is comparable to the proportions in the general population, where in the past three years $64 \%$ had undergone diabetic screening; and in the past one year $71 \%$ had undergone hypertensive screening, 61\% had undergone cholesterol screening, 28\% had undergone urine test and $16 \%$ had undergone some form of radiographic imaging. ${ }^{(5)}$ Allibone et al reported that among British GPs, 30\% of male GPs and 32\% of female GPs had their blood pressure checked in the past three years, $36 \%$ of male GPs and $49 \%$ of female GPs had undergone urine tests in the 
past three years, and $54 \%$ of male GPs and $76 \%$ of female GPs had chest radiography performed in the past three years. ${ }^{(4)}$

The present study was not without limitations. We recognise the inherent limitations of a cross-sectional survey. The survey was based on self-reported information regarding the PCPs' personal health, which may have been a sensitive topic. In addition, we were unable to verify the data obtained, which could have been subject to recall bias. Previous studies on local PCPs regarding other topics relied on postal survey, resulting in suboptimal response rates. ${ }^{(13)}$ Such a survey design could potentially select respondents with a strong positive or negative opinion about their health-seeking behaviour and skew the data in favour of extremes. We thus sought to optimise the participation rate by carrying out the survey in two phases, one of which was a web-based survey. Since most Singaporeans have Internet access and are savvy in its use, the web-based survey allowed PCPs to participate in the survey at any hour convenient to them. Anonymity of the participants was also maintained to increase the response rate. The present study's survey yielded the largest number of responses from PCPs, and as there is no reason to suggest that the PCPs who did not participate are intrinsically different, we believe that the findings of the present study (which had a response rate of $45 \%$ ) is representative of the local PCPs in general. The overall percentage of random missing data was $14 \%$. This was another shortcoming of the use of an anonymised survey - we were unable to address any incomplete administration of the questionnaires.

In conclusion, few PCPs in Singapore have undesirable habits such as smoking and alcohol abuse. However, PCPs can be more proactive in enhancing and maintaining their good health by obtaining immunisation against common infectious diseases and exercising regularly.

\section{ACKNOWLEDGEMENTS}

The authors would like to thank Mr Dennis Hu and Mr Chris Koh for their expertise in setting up the online survey, and the staff of the College of Family Physicians Singapore for their assistance in the postal survey.

\section{REFERENCES}

1. Schlicht SM, Gordon IR, Ball JR, Christie DG. Suicide and related deaths in Victorian doctors. Med J Aust 1990; 153:518-21.

2. Kay MP, Mitchell GK, Del Mar CB. Doctors do not adequately look after their own physical health. Med J Aust 2004; 181:368-70.

3. Health Promotion Board. Aim For 150 Minutes Of Physical Activity Every Week [online]. Available at: http://www.hpb.gov.sg/HOPPortal/healtharticle/10346. Accessed July 13, 2012.

4. Allibone A, Oakes D, Shannon HS. The health and health care of doctors. J R Coll Gen Pract 1981; 31:728-34.

5. Singapore Ministry of Health. Epidemiology and Disease Control Department. National Health Survey 2010. Singapore: Epidemiology and Disease Control Division, Ministry of Health, 2011.

6. Smith DR, Leggat PA. An international review of tobacco smoking in the medical profession: 1974-2004. BMC Public Health 2007; 7:115.

7. Josseran L, King G, Guilbert P, Davis J, Brucker G. Smoking by French general practitioners: behaviour, attitudes and practice. Eur J Public Health 2005; 15:33-8.

8. Christini $A B$, Shutt $K A$, Byers KE. Influenza vaccination rates and motivators among healthcare worker groups. Infect Control Hosp Epidemiol 2007; 28:171-7.

9. Singapore Ministry of Health. Top 4 Conditions of Polyclinic Attendances. Available at: http://www.moh.gov.sg/content/moh_web/home/statistics/ Health_Facts_Singapore/Top_4_Conditions_of_Polyclinic_Attendances. html. Accessed July 13, 2012.

10. Centers for Disease Control and Prevention. Seasonal Influenza (flu). Who Should Get Vaccinated Against Influenza. Available at: http://www.cdc. gov/flu/protect/whoshouldvax.htm. Accessed July 13, 2012.

11. Hofmann F, Ferracin C, Marsh G, Dumas R. Influenza vaccination of healthcare workers: a literature review of attitudes and beliefs. Infection 2006; 34:142-7.

12. Tapiainen T, Bar G, Schaad UB, Heininger U. Influenza vaccination among healthcare workers in a university children's hospital. Infect Control Hosp Epidemiol 2005; 26:855-8.

13. Chan R, Khoo L, Goh CL, Lam MS. A knowledge, attitudes, beliefs and practices (KABP) survey on HIV infection and AIDS among doctors and dental surgeons in Singapore. Ann Acad Med Singapore 1997; 26:581-7. 